\title{
Arbor
}

\section{El papel del Grupo Interuniversitario de Física Teórica (GIFT) en la FAE española}

\section{Manuel Asorey}

Arbor CLIX, 626 (Febrero 1998), 193-204 pp.

El Grupo Interuniversitario de Física Teórica constituyó durante casi veinticinco años una de las organizaciones científicas, no gubernamentales, más singulares del panorama científico español. El GIFT impulsó el desarrollo de la Física Teórica desde un estadio muy primitivo hasta las cotas más avanzadas del panorama científico internacional. El análisis de las actividades y estructura del GIFT revela algunas de las claves fundamentales para comprender este espectacular desarrollo. La existencia de una organización autónoma en ámbito de la Física Teórica propició una eficaz optimización de la utilización de los recursos y una homogeneización de los objetivos científicos y métodos de trabajo de los grupos de investigación, radicados en distintas universidades e institutos públicos de investigación. En este breve apunte se realiza, a la luz de los resultados obtenidos, un recorrido por la corta pero interesante historia del GIFT.

\section{Introducción}

Cualquier estudio sobre la evolución de la Física Teórica en España en el último tercio del siglo XX, no puede dejar de destacar el crucial papel desempeñado por el Grupo Interuniversitario de Física Teórica 
(GIFT) en el espectacular desarrollo de la misma. El GIFT actuó como fermento y catalizador de la vertiginosa carrera de los físicos teóricos españoles en su empeño por alcanzar los niveles científicos más avanzados de la Física Teórica contemporánea. El esfuerzo ha sido tan intenso que aún no ha habido tiempo para realizar un análisis histórico objetivo del mismo ${ }^{\dagger}$, aunque sin duda en un futuro no muy lejano los historiadores de la Ciencia abordarán el estudio profundo y riguroso de tan interesante fenómeno.

El objetivo de estas líneas se limita a la descripción de lo que el GIFT significó para el desarrollo de la Física Teórica en España y a la exposición de algunas reflexiones personales acerca de ciertos aspectos de tan singular agrupación. Por la propia naturaleza del estudio, deliberadamente las luces se destacarán sobre aquellas posibles sombras que sin duda también acompañaron al GIFT en su periplo.

\section{Los origenes del GIFT}

Al final de los años sesenta, en plena efervescencia cultural y política de las universidades españolas, surge la idea de impulsar la creación de una organización interuniversitaria que agrupe a los jóvenes físicos teóricos con la finalidad de impulsar el desarrollo de la Física Teórica en España. La idea había venido gestándose por un reducido grupo de físicos aglutinados entorno a la Junta de Energía Nuclear que, conscientes del enorme retraso que en dicha área España llevaba con respecto al resto de paises desarrollados, vislumbraron la posibilidad de recuperar gran parte del tiempo perdido aunando sus esfuerzos y reclamando de manera conjunta el apoyo de las instituciones oficiales.

En septiembre de 1968 se constituyó formalmente el Grupo Interuniversitario de Física Teórica (GIFT). Los objetivos iniciales del GIFT se centraron en impulsar el papel de la Física Teórica en el mundo académico universitario, potenciar la formación de jóvenes postgraduados, y estimular el desarrollo de la investigación en Física Teórica en España. Sus veintiocho miembros iniciales era doctores provenientes de las universidades de Barcelona, Madrid, Sevilla, Valencia, Valladolid y Zaragoza, el Consejo Superior de Investigaciones Científicas (CSIC)

\footnotetext{
† Recientemente, con motivo de la conmemoración del XXV aniversario de la fundación del GIFT, A. Galindo, quien fue su primer Director, realizó una revisión de los aspectos mas relevantes de su historia ${ }^{23}$.
} 


\section{El papel del Grupo Interuniversitario de Física Teórica}

y la Junta de Energía Nuclear (JEN), hoy reconvertida en el Centro de Investigaciones Medio Ambientales (CIEMAT).

La iniciativa se vió seriamente afectada por la retirada de España de la Organization Européenne pour la Recherche Nucleaire (CERN) en 1968. Este abandono, fundamentalmente motivado por razones económicas, se justificó políticamente enfatizando el escaso papel jugado hasta entonces por la industria y los científicos españoles en las actividades del CERN. Los argumentos utilizados para justificar la salida de España del CERN fueron aprovechados por la incipiente organización para reclamar un apoyo financiero institucional que remediase los males apuntados en el desarrollo de la Física de Altas Energías en España. Un acuerdo entre el Ministerio de Educación y Ciencia y el Ministerio de Industria, que estableció la concesión de una financiación institucional a través del Instituto de Estudios Nucleares (IEN) de la Junta de Energía Nuclear, fue decisivo para impulsar la actividad de la recién creada agrupación.

Con un presupuesto inicial de 16 millones de pesetas, en el año 1969 el GIFT estableció un programa de choque basado en una ambiciosa política de becas postdoctorales en centros extranjeros, que sería complementada con la financiación de estancias de investigación más cortas en los mismos y el apoyo a la participación de los miembros del GIFT en conferencias internacionales. Al mismo tiempo, el GIFT promovió la impartición de cursos de formación interuniversitarios para postgraduados e impulsó la convocatoria de un Coloquio anual de Física Teórica ${ }^{1}$ que enseguida se transformó en un Seminario Internacional de Física Teórica.

\section{La edad de oro}

Entre las características orgánicas más peculiares del GIFT como organización científica destaca el carácter colegiado de su dirección, constituida por un Director elegido directamente por los miembros y la Junta de Representantes de las distintas Secciones Locales. Las decisiones debían ser refrendadas en Asamblea General que se convocaba con periodicidad anual. La elección del Director debía realizarse cada dos años y el cargo era renovable solamente una vez. El carácter interuniversitario del GIFT, que incluye también a los investigadores del CSIC y de la JEN, estaba dictado por la estructura abiertamente descentralizada del mundo académico español. La dispersión geógrafica de los distintos grupos y la ausencia de un gran centro de referencia 


\section{Manuel Asorey}

condicionan la estructura de la agrupación. La única institución científica europea que guarda cierta similitud con la estructura del GIFT es el Istituto Nazionale di Fisica Nucleare italiano.

TABLA 1

\begin{tabular}{|c|c|c|c|}
\hline Año & $\begin{array}{l}\mathrm{n} \cdot{ }^{2} \text { miembros } \\
\text { (doctores) }\end{array}$ & $\begin{array}{l}\mathrm{n} \cdot{ }^{\circ} \text { miembros } \\
\text { asociados }\end{array}$ & $\begin{array}{l}\text { Presupuesto } \\
\text { (miles pts.) }\end{array}$ \\
\hline 1969 & 28 & 34 & 16.400 \\
\hline 1970 & 31 & 50 & 13.271 \\
\hline 1971 & 39 & 45 & 12.000 \\
\hline 1972 & 44 & 46 & 12.500 \\
\hline 1973 & 48 & 52 & 12.500 \\
\hline 1974 & 58 & 47 & 12.500 \\
\hline 1975 & 69 & 49 & 12.500 \\
\hline 1976 & 69 & 54 & 1.465 \\
\hline 1977 & 76 & 55 & 1.616 \\
\hline 1978 & 84 & 52 & 1.500 \\
\hline 1979 & 84 & 52 & 1.930 \\
\hline 1980 & 98 & 25 & 1.950 \\
\hline 1981 & 100 & 22 & 1.700 \\
\hline 1982 & 114 & 11 & 1.800 \\
\hline 1983 & 117 & - & 2.300 \\
\hline 1984 & 122 & - & 4.200 \\
\hline 1985 & 125 & - & 4.100 \\
\hline 1986 & 125 & - & 4.775 \\
\hline 1987 & 140 & - & 4.375 \\
\hline 1988 & 150 & - & - \\
\hline 1989 & 150 & - & - \\
\hline 1990 & 150 & - & - \\
\hline
\end{tabular}

Si exceptuamos los cinco primeros años, el presupuesto del GIFT ha sido modesto, especialmente si se compara con las importantes funciones que venía desempeñando tanto en la formación de jóvenes investigadores como en la promoción de la investigación y las relaciones internacionales. 


\section{El papel del Grupo Interuniversitario de Física Teórica}

Para ser elegido miembro del GIFT se exigía poseer el título de doctor, pero esta titulación no era suficiente. Se necesitaba además una experiencia investigadora propia que suele venir avalada por una estancia postdoctoral en el extranjero. Para incorporar a los investigadores que no eran doctores, se creó la figura del miembro asociado para el que los requisitos eran menos exigentes. Dicha categoría comenzó a extinguirse a principios de los años ochenta al suprimir la admisión de nuevos miembros asociados. El carácter de autoexigencia que el GIFT se impone sirve también de motivación y estímulo a los potenciales candidatos a incorporarse al mismo. Para ilustrar la disciplina y autocontrol del rigor y la calidad basta mencionar la implantación durante algún tiempo de una evaluación de la tarea realizada por los distintos miembros destinada a confirmar o no su adecuación a los objetivos de sus programas. Es difícil encontrar precedentes de una iniciativa similar en otros ámbitos científicos o académicos nacionales.

Durante los años que se mantuvo la financiación del IEN en sus términos iniciales (1969-1975) la actividad del GIFT se fortaleció en todos los frentes de su competencia, incrementando de forma acelerada el nivel científico de la Física Teórica española. El efecto inmediato de esta política científica se refleja en la duplicación del número de doctores y la quintuplicación del número de publicaciones por año. Estas últimas no sólo incrementan su número de forma considerable sino que cada vez aparecen en revistas internacionales de más prestigio.

Coincidiendo con la transición política, el desarrollo del GIFT recibe un duro golpe al no mantener el IEN su finaciación en los mismos términos que lo había venido haciendo hasta entonces. A partir de 1976 la financiación sólo se dirige a las actividades generales del GIFT (Cursos Interuniversitarios, Seminario Internacional y Convenios Internacionales). La ayuda institucional para las actividades investigadoras directas pasa a concederse directamente a los distintos grupos durante los años 1976 y 1977. En 1978 también desaparece este tipo de ayudas. Obviamente, con estos recortes desapareció la política de becas posdoctorales propias, aunque los distintos grupos siguieron promoviendo las estancias postdoctorales en el extranjero gracias a las becas concedidas por el MEC y diversas instituciones tanto nacionales como extranjeras.

A pesar del drástico cambio, el GIFT prosigue sus actividades organizando con entusiasmo los Cursos Interuniversitarios que siguen siendo fuente de formación para los nuevos doctores. También el Se- 
minario Internacional prosigue su rumbo aumentando su prestigio internacional con la participación de destacados físicos como invitados y la publicación de sus actas por prestigiosas editoriales. El número de estudiantes extranjeros que acuden a los mismos también aumenta considerablemente durante este período. Al mismo tiempo el GIFT financió parcialmente la organización de Congresos sectoriales tales como los Encuentros Relativistas, Topical Meetings on Nuclear Physics, Winter Meetings on Fundamental Physics y Sitges Schools on Statistical Physics.

Desde sus orígenes el GIFT promueve la colaboración internacional mediante convenios con distintos centros extranjeros. Merecen destacarse los establecidos con el International Center for Theoretical Physics de Trieste, el Centro Latino-Americano de Física de Brasil, la Universidad de Varsovia y la Universidad Autónoma de Méjico. En estos convenios se consiguen subvenciones para estancias de miembros del GIFT en dichos centros. También se establecen convenios para el intercambio de científicos españoles, franceses e ingleses a través la embajada de Francia y el British Council. Asimismo el Department of Physics de la New York State University de Stony Brook concedió dar prioridad a la aceptación de estudiantes de doctorado españoles presentados por el GIFT, con excelentes rendimientos científicos.

\section{Los años ochenta}

Con la llegada de la democracia, la política ciéntifica y universitaria sufre una inflexión crucial en su desarrollo que culmina en la creación de la Comisión Asesora de Investigación Científica y Técnica (CAICYT). El recién creado marco político abrió nuevos cauces de financiación para todas las áreas científicas. Los grupos investigadores pasaron a tener una finaciación directa. Una organización autónoma como era el GIFT difícilmente encajaba con las nuevas estructuras.

A pesar de ello, se consiguió un pequeño incremento en el presupuesto gracias a nuevas subvenciones provenientes del CSIC y de la CAICYT (actualmente denominada CICYT) dirigidas fundamentalmente a las actividades generales del GIFT. Resulta paradójico que con el boom del desarrollo científico español de los años ochenta, el GIFT fuese desapareciendo paulatinamente como protagonista del desarrollo de la actividad en Física Teórica. Los denodados esfuerzos de los distintos Directores del GIFT por conseguir una institucionalición del mismo, bien como un Instituto del Consejo de Investigaciones Científicas, bien como otro tipo de Organismo Científico, no tuvieron éxito y finalmente 


\section{El papel del Grupo Interuniversitario de Física Teórica}

se decidió la integración de los miembros del GIFT en el Grupo Especializado de Física Teórica de la Real Sociedad de Física (RSEF). El incremento de la financiación de la investigación por los nuevos cauces institucionales también contribuyó decisivamente a que el interés de muchos de sus miembros en la organización del GIFT disminuyese considerablmente. Asimismo, el aumento del tamaño de los distintos grupos produjo una mayor diversificación en los temas de interés que se reflejó en una gran dispersión en las actividades del GIFT, que ya se muestra incapaz para captar el interés de todos sus miembros. Con ello inicia el GIFT un período de decadencia que conducirá a su desaparición al comienzo de los años noventa.

\section{Hitos del GIFT}

Durante más de dos decadas el GIFT organizó cada año sin ninguna interrupción un Seminario Internacional, seleccionando con sumo cuidado tanto el tema central del mismo como los organizadores y conferenciantes invitados. El Seminario Internacional del GIFT pronto se convirtió por su calidad en un punto de referencia en Europa, lo que propició un incremento notable de la participación de estudiantes extranjeros. En consonancia con la elección del tema del seminario se programaban previamente cursillos interuniversitarios sobre el mismo, enfatizando su carácter fundamentalmente formativo para los miembros no doctores. Los resúmenes de la mayoría de estos Seminarios y Cursos Interuniversitarios aparecen en publicaciones científicas, inicialmente editadas por el propio Servicio de Publicaciones del GIFT, radicado en la Universidad de Zaragoza, y posteriormente por prestigiosas editoriales científicas ${ }^{2-22}$.

TABLA 2. INTERNATIONAL GIFT SEMINARS

I. Interactions of Particles and Nuclei, Director: P. Pascual, Valencia (1970)

Conferenciantes: M. Ericson, T.E.O. Ericson, O. Lofoed-Hansen, M. Rho, H.P.C. Rood y F. Scheck

Ii. Analytical Theory of the S Matrix, Director: F.J. Ynduráin, Madrid (1971) Conferenciantes: D. Atkinson, R.J. Eden, A. Donnachie, A. Martin y G. Wanders

III. Broken Symmetries, Director: A. Galindo, Madrid (1972)

Conferenciantes: N. Cabbibo. L. Michel. R.J. Oakes. B. Renner. R. Thom y B. Zumino 


\section{Manuel Asorey}

IV. Strong Interactions at High Energies, Organizadores: P. Pascual y R. Guardiola, Barcelona (1973)

Conferenciantes: L. Bertocchi, A.N. Diddens, R.J. Glauber, C. Hong-Ho, B. Schremp y F. Schremp

V. Some Topics in Weak Interactions, Director: A. Morales, Zaragoza (1974)

Conferenciantes: A. De Rújula, P. K. Kabir, W. Kummer, G. Marx, H. Pietschmann y D. Tadic

VI. Quantum Field Theory, Director: L.J. Boya, Jaca (1975)

Conferenciantes: J. Bell, S. Coleman, K. Osterwalder, K. Zymanzik, C. Jarlskog (Seminario: E. Santos)

VII. Phenomenology of Particle Physics, Director: A. Bramon, L' Escala (1976)

Conferenciantes: F.E. Close, J. Kuti, V.D. Nanopoulos, G. Preparata y F.M. Renard (Seminarios: J.L. Alonso, J. Bernabéu, A. Capella, M. Greco, R. Nuñez-Lagos, M. Ramón-Medrano y F.J. Ynduráin)

VIII. Topics in Quantum Field Theory and Gauge Theories, Director: J.A. de Azcárraga, Salamanca (1977)

Conferenciantes: P. Goddard, J. Wess, J. Zinn-Justin, A.J.G. Hey, J. C. Pati, A. de Rújula, R.H. Dalitz

IX. Nonlinear Problems in Theoretical Physics, Director: A.F. Rañada, Jaca (1978) Conferenciantes: A.O. Barut, I. Bialynicki-Birula, D. Levi, C. M. Dafermos y W. Strauss (Seminarios: M.R. Feix y C. Montes)

X. Quantum Chromodynamics, Organizadores: J.L. Alonso, J. Bernabeu, C. Pajares, R. Tarrach y F.J. Ynduráin, Jaca (1979)

Conferenciantes: J. Bartels, H. Fritzsch, H. Leutwyler, H. Lynch, E. de Rafael y C. Sachrajda

XI. Electroweak Theories, Organizadores: J. Bernabéu, P. Pascual y J. Sánchez-Guillén, Peníscola (1980)

Conferenciantes: E. de Rafael, K.J.F. Gaemers, C. Jarlskog, P. Minkowski y L. M. Sehgal (Seminars: J. Bernabéu y F.J. Ynduráin)

XII. Non-Perturbative Aspects of Quantum Field Theory, Organizadores: J. Julve y M. Ramón-Medrano, Sant Feliu de Guixols (1981)

Conferenciantes: P. Di Vecchia, J.L. Gervais, C.P. Korthals Altes, C. Rebbi, J. Zinn-Justin y M. Rasetti

XIII. $S U(3) \times S U(2) \times U(1)$ and Beyond, Organizadores: A. Ferrando, J. A. Grifols y A. Méndez, Masella (1982)

Conferenciantes: A. Ali, R. Barbieri, F. Barreiro, J. Bernabéu, F. Eisele, A. González-Arroyo, G. Marinelli, D.V. Nanopoulos, M. Quirós, G.G. Ross, L.R. Sulak, R. Tarrach y F.J. Ynduráin

XIV. Relativistic Astrophysics and Cosmology, Organizadores: X. Fustero y E. Verdaguer, Sant Feliu de Guixols (1983)

Conferenciantes: J. D. Barrow, M. J. Rees, S. T.C. Siklos, J. Silk, B.F. Schutz y M. Warner

XV. Supersymmetry, Supergravity and Related Topics, Organizadores: F. del Aguila, J.A. de Azcárraga y L.E. Ibáñez, Sant Feliu de Guixols (1984)

Conferenciantes: K.S. Stelle, T. Regge, G.W. Gibbons, M.J. Duff, D.Z. Freedman, S. Raby, G.G. Ross, P. Ramond y P.K. Townsend

XVI. New Perspectives in Quantum Field Theories, Organizadores: J. Abad, J.L. Alonso y M. Asorey, Jaca (1985)

Conferenciantes: B.A. Berg, P. Ginsparg, G. 't Hooft, C. Itzykson, P.K. Mitter y R. Stora. (Seminarios: A. Actor, I.G. Halliday y C.P. Korthals Altes) 


\section{El papel del Grupo Interuniversitario de Física Teórica}

XVII. Cosmology and Particle Physics, Organizadores: E. Álvarez, R. Domínguez Tenreiro, J.M. Ibán̄ez y M. Quirós, Peñíscola (1986)

Conferenciantes: J. Audouze, F. Englert, T.W.B. Kibble, E.W. Kolb, M. Turner, P. Sikivie y J. Silk

XVIII. Strings and Superstrings, Organizadores: J.R. Mittelbrunn, M. Ramón-Medrano y G. Sierra Rodero, El Escorial (1987)

Confenenciantes: O. Álvarez, L. Álvarez-Gaumé, D. Friedan, M. Green, D. Olive y S. Samuel (Seminarios: L.E. Ibáñez y H.J. de Vega)

XIX. Non perturbative aspects of the Standard Model, Organizadores: J. Abad, B. Gavela y A. González-Arroyo, Jaca (1988)

Conferenciantes: M. Creutz, P. Hasenfratz, E. de Rafael, A. Ukawa, G. Martinelli, A.J. Buras, O. Pène, C.P. Korthals Altes, y N. Parga. (Seminarios: A. Cruz y I.K. Kostov)

XX. Integrability and Quantization, Organizadores: J.F. Carin̄ena, M. Fernández-Rañada y A. Ibort, Jaca (1989)

Confereciantes: O. Babelon, B. Dubrovin, K. Gawedzki, M. Henneaux, R. Jackiw, G. Marmo, A. Pressley y H.J. de Vega

XXI. Precision Tests of the Standard Model at High Energy Colliders, Organizadores: F. del Aguila, A. Méndez y A. Ruiz, Santander (1990)

Conferenciantes: W.F.L. Hollik, R. Kleiss, W.J. Stirling, T. Sjöstrand, C. Verzegnassi, Z. Kunszt, G.L. Klane, P.J. Dornan, D. Treille, M. Martínez, J. Marco, N. Colino y T. Kawamoto

XXII. Quantum Gravity and Cosmology, Organizadores: J. Pérez Mercader, J. Sola y E. Verdaguer, San Feliu de Guixols (1991)

Conferenciantes: E. Álvarez, P. Mazur, L. Smolin, W. Zurek, R. Laflame, C. Wetterich y J. Barrow

XXIII. Integrable Systems, Quantum Groups, and Quantum Field Theories, Organizadores: L.A. Ibort y M.A. Rodríguez, Salamanca (1992)

Conferenciantes: L.D. Faddeev, C. Gómez, H. Ruegg, D.S. Freed, C.J. Isham, R. Jackiw P.K.

Townsend, G. Sierra y P. Winternitz (Seminarios: A. Bohm, M. Gadella y L. Vinet)

Los veintitrés seminarios internacionales del GIFT abordaron los temas centrales de la Física Teórica contemporánea. Entre los conferenciantes se encuentran muchos de los científicos más influyentes del momento.

Durante todos estos años la dirección del GIFT ha estado en manos miembros de reconocido prestigio como son A. Galindo, P. Pascual, A. Rañada, R. Pascual, J. Bernabéu, J.L. Alonso y J.L. Sánchez Gómez. Una labor que también merece destacarse es la realizada por el primer secretario científico y responsable del servicio de publicaciones A. Morales.

El nivel científico alcanzado en tan corto espacio de tiempo es impresionante. El número de publicaciones científicas en las mejores revistas internacionales y sus citas no cesan de aumentar. En las conferencias internacionales de las distintas especialidades también destacan un número importante de invitados españoles. Del mismo modo, cada vez aparecen con más frecuencia físicos teóricos españoles en los comités organizadores de dichas conferencias así como en los consejos editoriales de las revistas más prestigiosas. Como símbolo de 
esta creciente influencia internacional baste mencionar la presencia de dos miembros del GIFT entre el reducido núcleo de miembros permanentes del grupo teórico del CERN, uno de los cuales es su director actual. También miembros del GIFT ocupan puestos de liderazgo científico en institutos de investigación de Francia (Orsay y Marsella) y EE.UU. (Oak Ridge). Aunque todos estos éxitos son fundamentalmente debidos a los méritos y calidad de los respectivos miembros involucrados, tampoco debe menospreciarse el papel jugado por el GIFT en la génesis de los mismos. El apoyo externo prestado por estos científicos, integrados en instituciones internacionales, a las iniciativas del GIFT ha sido siempre fundamental.

Finalmente, cabe destacar también el papel de los miembros del GIFT en el apoyo a la reincorporación de España al CERN, objetivo que se logró en 1983 y en la promoción de los grupos experimentales en Física de Altas Energías en las distintas Universidades españolas, cumpliendo así su compromiso fundacional vinculado al desarrollo de las Altas Energías en todas sus facetas. Esta actitud, no siempre bien comprendida, demuestra la coherencia, objetividad y espíritu científico que caracterizó al GIFT.

\section{El legado del GIFT}

Gracias al GIFT la Física Teórica se adelantó en más de una década al desarrollo de la media de las especialidades científicas y eso hizo que se colocase en una situación de privilegio al inicio de la gran expansión científica de los ochenta. Además del nutrido grupo de investigadores y profesores de alta cualificación científica distribuidos por toda la geografía nacional e internacional, el legado del GIFT a la comunidad científica española incluye la existencia de vínculos muy estrechos entre los distintos grupos de investigación que se reflejan en una gran homogeneización en los criterios, objetivos y metodología, utilizados en el desarrollo de las diferentes líneas de investigación. En concreto, el interés inicial común en la Física de Altas Energías se mantiene conjuntamente con aspectos fundamentales de Física Matemática, Física Nuclear, Mecánica Estadística y Gravitación.

El GIFT ha gozado de un gran prestigio tanto nacional como internacional que todavía perdura hoy, no sólo en la memoria de un gran número de científicos, sino también en las bibliotecas, hemerotecas y bases de datos electrónicos más consultadas. El éxito del GIFT no siempre ha sido bien entendido por la comunidad ciéntifica nacional. 
La extensión a las demás especialidades de los rigurosos criterios de valoración de la labor académica e investigadora, promovidos por miembros del GIFT, ha contribuido a crear tensiones en ciertos medios académicos lo que ha generado finalmente una cierta atmósfera hostil a lo que el GIFT y la Física Teórica representan.

La desaparición del GIFT coincide con la etapa de madurez de la Física Teórica en España, pero algunas funciones que el GIFT desarrollaba todavía son imprescindibles y de hecho no han desaparecido con el mismo. En particular, los cursos de formación de doctores, las escuelas internacionales y los encuentros nacionales entre los distintos grupos parecen resurgir con fuerza. En cualquier caso, veinticinco años después de la constitución del GIFT las necesidades de la Física Teórica en España han cambiado radicalmente, y esencialmente coinciden con las que afectan a la especialidad en los demás países desarrollados. El futuro prometedor que se abre a la misma no debe ocultar los enormes esfuerzos realizados hasta ahora y que el GIFT ha sabido canalizar con gran éxito, economía de medios y eficacia. Sin duda el espíritu que el GIFT impregnó a la Física Teórica en la España del último tercio del siglo $\mathrm{XX}$ es una fuente de la vitalidad y pujanza con que ésta aborda el comienzo del nuevo milenio.

\section{Blbliografía}

1 Proceedings of the First Coloquium in Theoretical Physics: E. DE RAFAEL, M. Gourdin y M. JACOB, GIFT Sci. Inf. Service, Zaragoza (1969).

2 Interactions of Particles and Nuclei, Ed. P. PAScual. GIFT Sci. Inf. Service, Zaragoza (1970).

${ }^{3}$ Analytical Theory of the S Matrix: Ed. F.J. YNDURAin. GIFT Sci. Inf. Service, Zaragoza (1971).

${ }^{4}$ Broken Symmetries, Ed. A. Galindo. GIFT Sci. Inf. Service, Zaragoza (1972).

5 Strong Interactions at High Energies, Eds. P. PASCUAL y R. GUARDIOLA. GIFT Sci. Inf. Service, Zaragoza (1973).

${ }^{6}$ Some Topics in Weak Interactions, Ed. A. Morales. GIFT Sci. Inf. Service, Zaragoza (1974).

7 Phenomenology of Particle Physics, Ed. A. Bramon, GIFT Sci. Inf. Service, Zaragoza (1976).

8 Topics in Quantum Field Theory and Gauge Theories, Ed. J. A. de AzCÁRRAGA, Lecture Notes in Physics 77, Springer-Verlag, Berlin (1978).

9 Nonlinear Problems in Theoretical Physics, Ed. A. F. RAÑADA, Lecture Notes in Physics 98, Springer-Verlag, Berlin (1979).

${ }_{10}$ Quantum Chromodynamics, Eds. J. L. ALONSO y R. TARRACH, Lecture Notes in Physics 118, Springer-Verlag, Berlin (1980). 
11 Non-Perturbative Aspects of Quantum Field Theory, Eds. J. Julve y M. RA. MÓN-MEDRANO. World Sci., Singapore (1982).

$12 S U(3) \times S U(2) x U(1)$ and Beyond, Eds. by A. Ferrando, J. A. Grifols y A. MÉndez. World Sci., Singapore (1983).

13 Relativistic Astrophysics and Cosmology, Eds. X. Fustero y E. Verdaguer. World Sci., Singapore (1984).

14 Supersymmetry, Supergravity and Related Topics, Eds. F. DEL AGUILA, J. A. de Azcárraga y L. E. Ibáñez. World Sci., Singapore (1985).

15 New Perspectives in Quantum Field Theories, Eds. J. ABAD, M. ASOREy y A. CRuz. World Sci., Singapore (1986).

16 Cosmology and Particle Physics, Eds. E. Álvarez, R. Domínguez Tenreiro, J. M. IBÁṄEZ y M. Quirós. World Scientic, (1987).

17 Strings and Superstrings, Eds. J. R. MitTElbrunN, M. Ramón-MEdRano y G. SIERRA RODERO. World Sci., Singapore (1988).

18 Non perturbative Aspects of the Standard Model, Eds. J. ABAD, B. Gavela y A. González-Arroyo. Nucl. Phys. B (Proc. Suppl.) 10 A (1989).

19 Integrability and Quantization, Eds. M. ASOREY, J. F. CARIÑENA y A. IBORT, Nucl. Phys. B (Proc. Suppl.) 18 A (1990).

20 Precision Tests of the Standard Model at High Energy Colliders, Eds. F. DEL Aguila, A. Méndez y A. Ruiz. World Sci., Singapore (1991).

21 Quantum Gravity and Cosmology, Eds. J. PÉrez MerCader, J. Sola y E. VerDAGUER. World Sci., Singapore (1992).

22 Integrable Systems, Quantum Groups, and Quantum Field Theories. Eds. L. A. Ibort y M. A. Rodríguez. World Sci., Singapore (1993).

23 A. Galindo, Revista Española de Física, 8 (1994) 12-17. 